\title{
Evolving Rules for a Self-Organizing Finite Element Mesh Generation Algorithm
}

\author{
A. E. Langham \\ Department of Computer Science \\ University of Wales Swansea \\ Swansea, SA2 8PP, U.K. \\ csjim@swan.ac.uk
}

\author{
P. W. Grant \\ Department of Computer Science \\ University of Wales Swansea \\ Swansea, SA2 8PP, U.K. \\ p.w.grant@swan.ac.uk
}

\begin{abstract}
Using only simple stimuli from the local environment to coordinate the activity of the group, social insects such as termites build highly complex structures. This was discovered by Grassé [1] and is known as stigmergy . We employ such a distributed approach for meshing finite element domains. In two dimensions, a triangular mesh is a discretization of a domain into a set of approximately equilateral triangles. The finite element method can then be used to solve fluid flow problems at the vertices in discrete time. The size of the triangles is related to rate of change of the solution values and hence meshing is a complex task. We use a Genetic Algorithm to evolve a set of rules which can be used by a colony of termite-like agents to mesh an arbitrary domain. Work on building activity of swarms has shown that stigmergic algorithms can produce more complex structures than sequential algorithms which are dependent on past building activity. We compare our stigmergic algorithm with a similar sequential algorithm. Whilst the sequential algorithm generalizes poorly to new domains, our stigmergic approach generalizes well and produces better quality meshes on the examples considered. In this approach, meshing is in response to conditions in the local environment, hence changing conditions can easily be accommodated, making it particularly useful for applications such as injection moulding where the mesh must 'grow' with the domain.
\end{abstract}

\section{Introduction.}

Meshing involves discretizing a geometrical domain into a set of elements, usually triangles in two dimensional problems. Such elements are used in approximate engineering techniques such as the Finite Element Method, which solve, for example, fluid flow problems by approximating a solution at the nodes or vertices of each element. To produce an accurate solution the domain must be discretized with elements of the appropriate size and shape. Ideally, elements should be close to equilateral, relatively small in places where the solution values are changing rapidly (around holes for example) and relatively large where the rate of change is small. The required size of elements is defined by a domain specific density function.

The approach in this paper is to simulate a group of mobile termite-like agents which collectively construct a mesh by distributing the task. The Algorithm is self-organizing. It displays an emergent structure through the interaction of a population of agents. Here agents interact indirectly through the environment - there is no central control mechanism, the swarm is coordinated solely by the structure of the mesh.

The success of such an approach can be seen in natural systems such as insect colonies. In particular wasps and termites build highly complex structures using only simple interactions with their environment and honey bees carry out a variety of Three Dimensional meshing when building their honey combs. Bonabeau et al. [2] investigated the building behaviour of artificial agents on a lattice capable of depositing or removing bricks according to the state of the local environment. It was found that with very simple rules, the agents could build very complex structures in both two and three dimensions, many mimicking those seen in nature.

Deneubourg et al. [3] investigated similar behaviour on a lattice and compared a Stigmergic Algorithm with a Sequential One. The behaviour of an agent is affected by its past activities in the Sequential Algorithm whereas in the Stigmergic Algorithm it is only affected by the state of the local algorithm. The Stigmergic Algorithm was found to be more efficient (i.e. building rate per agent is higher) as the colony size increases and also capable of producing more complex structures.

A Sequential Algorithm was used by Saitou and Jakiela [4] to generate meshes using Meitotic Cell Division to produce elements. Cells are grown from an initial line segment by moving a grow point perpendicularly from the a point on the base until an element of the correct size is formed. Once grown, a cell can then reproduce on one of its two new sides in a similar fashion. After Growth, a cell can be merged with another cell by moving the grow point to a corner of another cell. The algorithm starts with a root cell on the boundary and grows recursively left and then right until all cells are dead, i.e. cannot grow. Parameters governing the position of the growpoint on the base and height of the cell are evolved using a set of rules which are dependent on the distance to the nearest boundary or element in eight directions. This can produce many poorly shaped and badly connected elements especially around the boundaries. Also the rules evolved for use on one domain do not generalize to another domain. Each domain needs to have a new set of rules evolved.

Inspired by the work of Deneubourg et al. [3] on mod- 
elling the construction of architectures of social insects seen in nature, our approach uses information in the local environment so that the rule set can generalize better to mesh a new domain. Furthermore, building activity is not dependent on past activity only on the state of the mesh in the local environment. This adds greater flexibility as do the addition of new types of building behaviour such as deleting elements which are badly shaped or connected. Implementation can use either probabilistic rules, which give a probability of a particular action occurring, or use deterministic rules, which give one possible action given the state of the local environment. Both types of rules are investigated and the quality of the mesh and generalization to new domains is compared.

\section{The Method}

A domain $D$ is taken as a 2-Dimensional polygon with a density function $d$ determining the approximate required size of elements at a given point in the domain. The domain is equivalent to the nest environment for the termite colony. It is required to mesh the domain with elements of the desired size and shape each connected ideally to six other elements. Because the value of a density function can vary greatly across a domain, the area describing the local environment and all distances used in the building operations are related to the density function.

\subsection{Building operations}

There are various operations, and combinations of operations, which can be performed. The basic building block is element growth which is similar to the cell growth mechanism used by Saitou and Jakiela [4]. Here growth always starts from the midpoint of the base segment, $\boldsymbol{x}$, and the grow point is moved perpendicular to the base segment until the maximum perpendicular height $h$ is reached, the new element touches another element or the domain boundary (Figure 1). The perpendicular height of the element $h$ is calculated as follows: $h=0.75 *\left(d_{h}+e_{h}\right) / 2.0+0.25 * d_{h}$ where $d_{h}$ is the desired height calculated from the density function $d$ and the length of the base segment $b, d_{h}=1.0 /(d(\mathbf{x}) * b)$ and $e_{h}=b * \sqrt{3.0} / 2.0$ which corresponds to the height for an equilateral triangle with base length $b$. An element can grow from the right, left or base segment. This segment becomes the base segment for the new element. The three growth operations are denoted as follows $G_{L}$ for grow left, $G_{R}$ for grow right and $G_{B}$ for grow from the base. After growth, an element can merge with the point of another by moving the growth point to this point if the distance to this point is less than $0.5 * d_{h}$ and the merge does not cause an overlap with other elements.

Morph and Delete are dependent on the average fitness of the current element and all elements connected to the grow point of that element. This average fitness is called the local average $l_{a v}=0.6 * l_{\text {shape }}+0.4 * l_{\text {size }}$ and is a measure of the average size and shape of each element. $l_{\text {shape }}$ is the

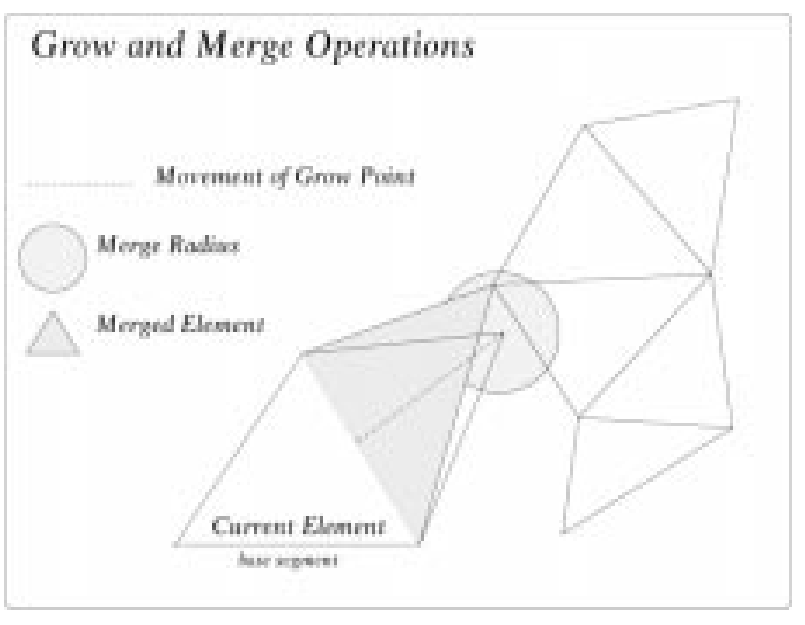

Figure 1: Grow Operation

average fitness for the shape of each connected element, and $l_{\text {size }}$ is the average fitness for the size of the connected elements. $l_{\text {shape }}$ and $l_{\text {size }}$ are defined in section 2.4 .1 for a set of $e$ elements, where $e$ is the number of elements incident on the grow point of the current element. The Morph operation $M_{\boldsymbol{A}}$ tries to improve the local average $l_{a v}$ by moving the grow point of the current element. This point is moved randomly within a small radius until a position is found which increases $l_{a v}$ or 10 attempts have been made without success. When the point is on a boundary, morph moves that point within the same distance but only along the line segment the point is currently on. The Delete operation $\boldsymbol{D}_{\boldsymbol{A}}$ deletes all connected elements and the current element if the local average is below a given level, (in our case empirically if $l_{a v}<0.7$ ). This is followed by the Jump operation $\boldsymbol{J}$ which moves to another element making the new element the current element.

Badly connected meshes occur when other elements are too close but not joined, hence preventing growth. When a node is within $0.3 * d_{h}$ of the centre point of any side of an element it is called a hanging node (Figure 2) and a special operation is used, Hanging Node Delete, $\boldsymbol{H}_{\boldsymbol{D}}$ which deletes all elements containing the hanging node and then grows from the side of the current element where the hanging node was found. As with any grow operation this is followed by a merge which tries to merge with another element or the boundary and is only successful if no overlap is produced between elements.

To stop small elements appearing on the boundary, after a morph or grow operation, a Join operation is used to merge two elements into one larger element. Morph can produce long thin elements as it may make one element worse in order to improve the fitness of the rest. Grow can also produce small elements if there is not enough space to grow an element of the desired size. Hence such elements can be joined into one element if the size of the element concerned is less than $0.3 * d_{a}$, where $d_{a}=1 /(2 d(\mathbf{x}))$ the desired area as defined by Saitou and Jakiela [4]. Join can only occur if two 


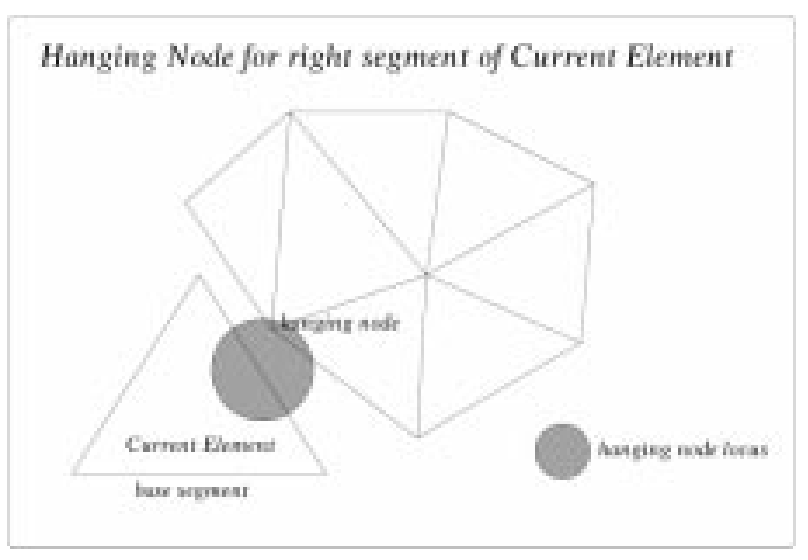

Figure 2: Hanging Nodes

elements both share a line segment which is not on a boundary line segment and both have another line segment which is on the same boundary line segment.

\subsection{Main algorithm}

The main algorithm consists of three phases:

- Boundary Growth,

- Inner Growth and

- Spice Up.

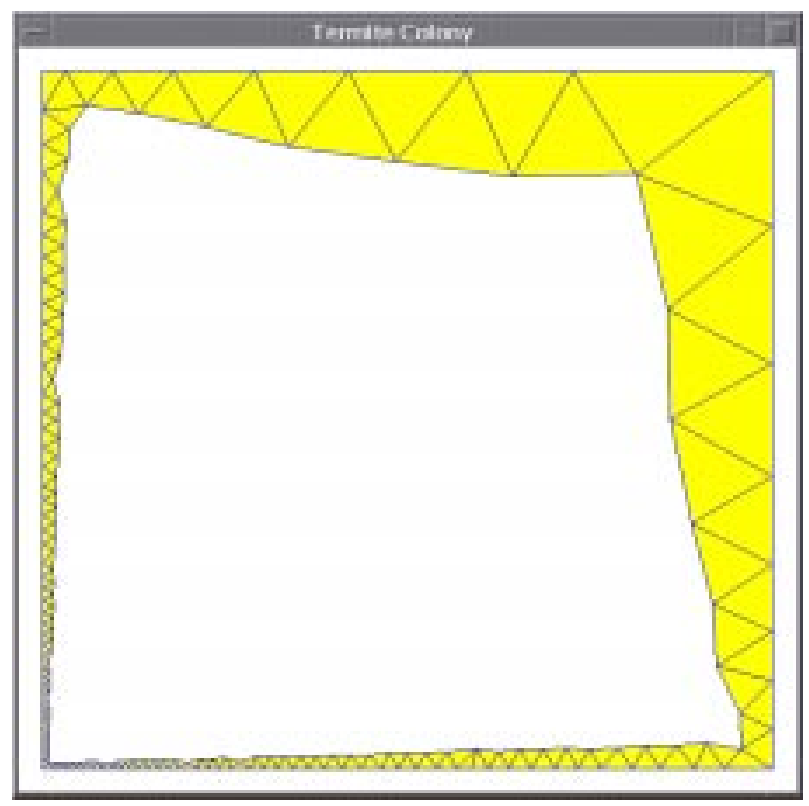

Figure 3: Boundary Growth

Boundary Growth and Inner Growth use exactly the same rule set but during the Boundary Growth phase (Figure 3) all Grow operations are restricted to element line segments with at least one node on a boundary line segment. In contrast, in the Inner Growth phase, Grow operations can be attempted from any element line segment. This produces well connected elements along the boundary which are difficult to produce without the Boundary Growth phase.

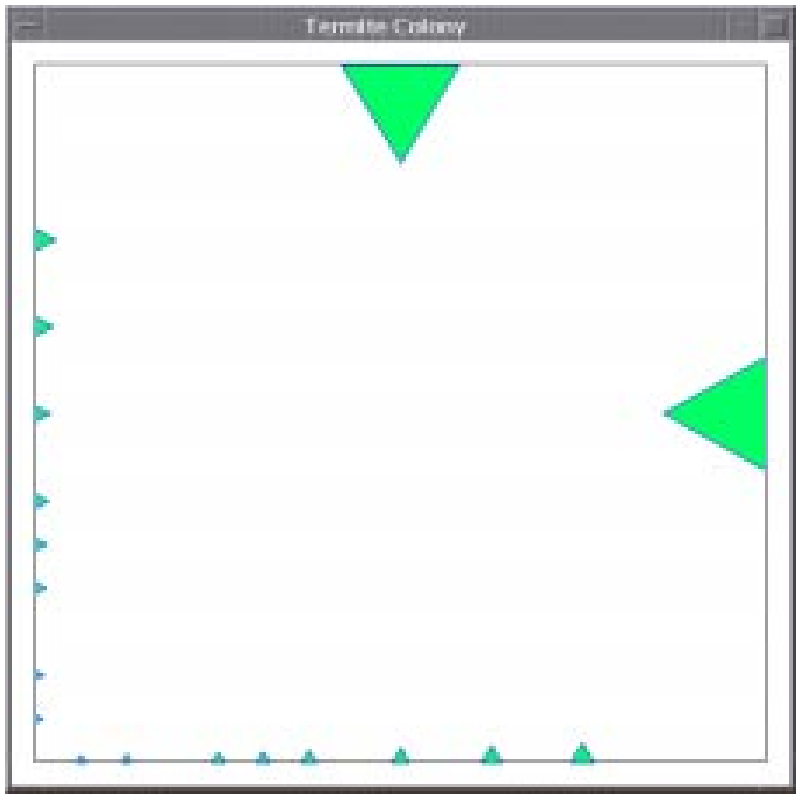

Figure 4: Roots Phase

Growth begins from root elements, which are placed recursively on each line segment of the boundary (Figure 4) as follows. A root is placed at the midpoint of each line segment. Roots are then placed recursively at the midpoints of the left and right segments, if the density function value is less than the value at the nearest root and the distance to the nearest root is greater that $3.0 * d_{h}$ of the midpoint of the current segment. Otherwise, if the density is greater than or equal to that at the nearest root, a new root is placed in the midpoint of this segment if the distance to the nearest root is less than $3.0 * d_{h}$ of the midpoint of the current segment.

Spice Up is used to finish off the mesh if the whole area has not been covered or a hanging node has not been removed. It repeatedly removes hanging nodes and forces elements to grow if possible until the whole domain is filled. The algorithm then jumps to random elements calling Morph to improve the local area fitness of elements. Spice up is not used during evolution of the rule set only when running on an arbitrary domain to make the method more efficient as it can be relatively time consuming to fill in the last one percent of the area.

To simulate the activity of a colony of termites on a sequential computer, the algorithm uses the Jump operation which can jump to any element in the mesh not just in the local area. This simulates the activity of many termites at different points in the domain each carrying out building operations. However to make mesh generation more efficient, Jump is biased towards elements which can grow. As the proportion of elements which can grow decreases, Jump is more 
likely to move to an element which can grow. This greatly increases the speed at which the mesh can be generated as a random Jump would make it difficult to find the last few elements which are able to grow. Jump either moves to a random element which can grow with probability $P_{J a c t}$ or a random element which cannot grow. This decision is related to the proportion of elements which are currently active or able to grow $p_{\text {act }}$ as follows

$$
P_{\text {Jact }}= \begin{cases}0.0 & \text { if } p_{a c t}=0 \\ 0.95 & \text { if } p_{a c t} \leq 0.05 \\ 0.90 & \text { if } p_{\text {act }} \leq 0.10 \\ 0.70 & \text { if } p_{\text {act }} \leq 0.30 \\ 0.50 & \text { otherwise }\end{cases}
$$

Each phase has a termination criterion. BoundaryGrowth terminates when less than 0.05 of the elements can grow along the boundary. Inner Growth terminates when less than 0.01 of the elements are active or can grow. Spice Up is then run which terminates when all elements are inactive and cannot grow.

\subsection{Rule format}

Rules are of the form if condition then action. The condition part relates to information in the local environment (Figure 5) and has seven components. The action part relates to a building operation or set of possible operations with associated probabilities. The first three fields of the condition relate to the state of each line segment of the current element, representing whether it is possible to grow off the left right and base segments. These fields are denoted by $\mathrm{Can}_{g_{l}}, \mathrm{Can}_{g_{r}}$, $\mathrm{Can}_{g_{b}}$, for can grow left, can grow right, can grow off base and take values 1 or 0 for true and false. The fourth field represents the local area fitness. If $l_{a v}$ is less than 0.75 it takes the value 1 for true, otherwise it has value 0 for false. This field is denoted by $\operatorname{Can}_{d_{a}}$ The fifth field is also boolean and is true if there is a hanging node and false otherwise. It is denoted by $I s_{h n}$. The last two fields can take integer values in $[0 . .3] . N_{c}$ is a measure of connectedness depending on $n_{c}$, the number of elements connected to the grow point of the current element given by

$$
N_{c}= \begin{cases}0 & \text { if } n_{c}=1 \\ 1 & \text { if } n_{c}=2,3,4 \\ 2 & \text { if } n_{c}=5,6,7 \\ 3 & \text { if } n_{c} \geq 8\end{cases}
$$

and $L_{\text {act }}$ measures the proportion of elements in the local area which are active (i.e. can grow) depending on $l_{\text {act }}$, the proportion of elements which are active in the local environment given by

$$
L_{a c t}= \begin{cases}3 & \text { if } l_{\text {act }} \leq 0.1 \\ 2 & \text { if } l_{\text {act }} \leq 0.2 \\ 1 & \text { if } l_{\text {act }} \leq 0.5 \\ 0 & \text { otherwise }\end{cases}
$$

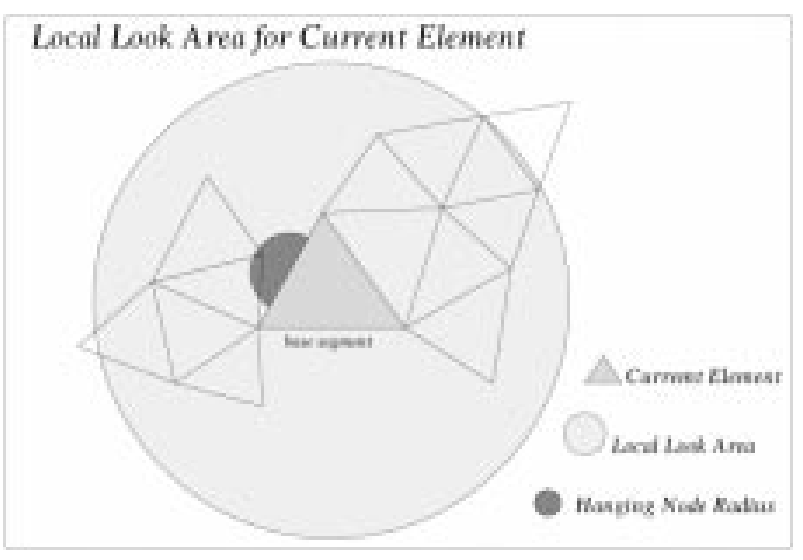

Figure 5: Local Look Area

Before choosing a rule, the algorithm finds the current values of all fields in the condition and matches this against the condition part of each rule. The rule with the most matches is chosen. If there is more than one such rule, then the first rule is chosen. Once a rule has been chosen the associated action is invoked. Actions can be either deterministic, relating to a single operation, or probabilistic giving a probability distribution of each possible action occurring.

\section{Deterministic Rule}

if $\left(\operatorname{Can}_{g_{l}}=b_{1}, \operatorname{Can}_{g_{r}}=b_{2}, \operatorname{Can}_{g_{b}}=b_{3}, \operatorname{Can}_{d}=b_{4}\right.$, $\left.I s_{h n}=b_{5}, N_{c}=n_{c}, L_{a c t}=n_{a}\right)$ then (action)

where, action $\in\left\{G_{L}, G_{R}, G_{B}, D, M, H\right\}$

$n_{c}, n_{a}$ are integers in [0..3], $b_{i} \in\{$ true, false $\}, i=$ $1, \ldots, 5$.

\section{Probabilistic Rule}

if $\left(\operatorname{Can}_{g_{l}}=b_{1}, \operatorname{Can}_{g_{r}}=b_{2}, \operatorname{Can}_{g_{b}}=b_{3}, \operatorname{Can}_{d}=b_{4}\right.$, $\left.I s_{h}=b_{5}, N_{c}=n_{c}, L_{a c t}=n_{a}\right)$

then $\left(P_{G_{L}}=p_{0}, P_{G_{R}}=p_{1}, P_{G_{B}}=p_{2}, P_{D}=p_{3}\right.$,

$\left.P_{M}=p_{4}, P_{H}=p_{5}, P_{J}=p_{6}\right)$

where the probabilities $p_{0} \ldots p_{6}$ represent the operations 0 to 6 as described in the deterministic rule and sum to 1.0 making a distribution. $n_{c}, n_{a}$ are integers on [0..3] and $b_{i} \in\{$ true, false $\}, i=1, \ldots, 5$.

\subsection{Evolving the rule set}

A Genetic Algorithm [5, 6] is used to evolve a rule set which can mesh the test domain according to the fitness function given below. Each rule set consists of $R_{N}$ rules. Each rule is represented by a bit string. This choice was originally made to facilitate crossover at the rule level. However, this was found too disruptive, so crossover at the rule set level was adopted instead, although mutation is still at the rule level. It would therefore be equally acceptable to operate directly on the rule sets and avoid an explicit bit string encoding. The condition part needs 9 bits, the deterministic action 3 bits and the probabilistic action 49 bits as each action is represented by 7 bits. The distribution can be found by dividing each 
number by the total sum of all numbers. A population of $P_{N}$ rule sets are used. All rules are randomly initialized. However, they are made consistent so that the condition fields do not conflict with the action or set of probable actions. For example if, as a premise, $\operatorname{Can}_{G_{L}}$ is false then $G_{L}$ is not allowed as a possible action. For deterministic rules the action bit string is repeatedly randomly generated until a permissible action is produced. For probabilistic actions, the bits representing an action which is not allowed are all set to zero. If all bits are set to zero in the action string another rule is generated.

In the sample executions, $R_{N}=50$ and $P_{N}=50$ and each rule set is run for 800 time steps on the square domain. Each time step represents one action which is derived by matching the state of the local environment against the condition part of each rule. The first rule with the greatest number of matches is invoked. If less than 5 matches are found, a random rule is invoked. If an action is unsuccessful the Jump operation is used to stop the algorithm from sticking. The first 200 time steps are used for Boundary Growth and the remaining 600 for Inside Growth. After 800 time steps the fitness of each mesh produced is calculated.

\subsubsection{Fitness function}

The fitness function $f$ has five components $f_{s}, f_{c}, f_{a}, f_{h n}$ and $f_{t}$ which are combined as a weighted average:

$$
f=\left(f_{s}+f_{c}+f_{h n}+2\left(f_{a}+f_{t}\right)\right) / 7
$$

Each of the components are described below:

$f_{s}$ is the average between the shape and size metrics. It is similar to that used in the Morph operation. It is defined as follows,

$$
\begin{aligned}
f_{s} & =0.4 * f_{\text {size }}+0.6 * f_{\text {shape }}, \\
f_{\text {size }} & =\sum_{i=1}^{e}\left(1-\left|1-\frac{a}{d_{a}}\right|\right) / e \\
f_{\text {shape }} & =\sum_{i=1}^{e} \sum_{j=1}^{3}\left(1-\left|1-\frac{\cos \theta_{j}}{0.5}\right|\right) / 3 e
\end{aligned}
$$

where $e$ is the number of elements, $a$ is the area of an element and $d_{a}$ is the desired area of an element and $\theta_{j}, j \in\{1,2,3\}$ represents the internal angles of an element. This is similar to the fitness measures defined by Saitou and Jakiela [4].

$f_{c}$ is the average connectivity of nodes. All nodes should ideally belong to six elements except boundary nodes which should in general be connected to three other elements.

$$
f_{c}=\sum_{i=1}^{n}\left(1-\left|\frac{n_{c}-d_{n_{c}}}{d_{n_{c}}}\right|\right) / e
$$

where $n$ is the number of nodes, $n_{c}$ is the number of elements connected to each node, and $d_{n_{c}}$ is the desired number of connected elements. $f_{a}$ is related to the proportion of the domain area covered by elements. If this proportion is less than 0.75 then $f_{a}$ is given the value zero. Hence,

$$
f_{a}= \begin{cases}\frac{p_{\text {area }}-0.75 * D_{\text {area }}}{0.25 * D_{\text {area }}} & \text { if } p_{\text {area }}>0.75 * D_{\text {area }} \\ 0 & \text { otherwise }\end{cases}
$$

where $p_{\text {area }}$ is the proportion of the domain area covered by elements and $D_{\text {area }}$ is the total area of the domain $D$.

$f_{h n}$ is related to the proportion of elements which have a hanging node on one of the growth segments. If this proportion is less than 0.03 then $f_{h n}$ is given a value of zero. Hence

$$
f_{h n}= \begin{cases}\frac{\left(1-p_{h n}\right)-0.97 * e}{0.03 * e} & \text { if } p_{h n}<0.03 \\ 0 & \text { otherwise }\end{cases}
$$

where $p_{h n}$ is the proportion of elements with hanging nodes and $e$ is the total number of elements.

$f_{t}$ is the time taken to reduce the proportion of elements which are active to 0.05 i.e $p_{\text {act }}<=0.05$. If this occurs within a minimum time $t_{\text {min }}$ of 500 time steps then $f_{t}$ takes a value of 1.0. Otherwise $f_{t}$ is indirectly proportional to the time taken $t_{\text {end }}$ to reach $p_{\text {act }}<=0.05$. Hence

$$
f_{t}= \begin{cases}1-\left(\frac{t_{\text {end }}-t_{\min }}{t_{\max }-t_{\min }}\right) & \text { if } t_{\text {end }} \geq 500 \\ 1.0 & \text { otherwise }\end{cases}
$$

Here $t_{\max }=800$ time steps.

\subsubsection{Reproduction operators}

A new generation of rule sets is produced using Crossover on the existing population. Two members are chosen probabilistically using a distribution function proportional to the fitness of each rule set. The probability of Crossover occurring is 0.9 , otherwise the first member is copied to the new population. Crossover between two rule sets takes place at the rule level. Crossover produces a new rule set by taking $n_{1}$ rules from rule set 1 , where $n_{1}$ is a random number from [1..N], and $N-n_{1}$ from rule set 2 . The rules which fire are chosen first with equal probability and if there are not enough a non-firing rule is chosen, each with equal probability. Each rule can only be chosen once to avoid unnecessary duplication. Mutation has a probability of 0.1 of changing the value in a field of the rule bit string. If a field which is represented by more than one bit is to be mutated a random bit in that field is flipped. After Crossover and Mutation the rules are made consistent as described earlier. The position of rules in each new rule set is then randomly reallocated so that the rules chosen from rule set 1 do not dominate in the matching process.

\section{Results}

The evolved rule sets are run on the test domain $D_{t}$ for 800 time steps, the resulting meshes are shown in Figures 6 and 7. The rule sets evolved are then tested on three new domains 


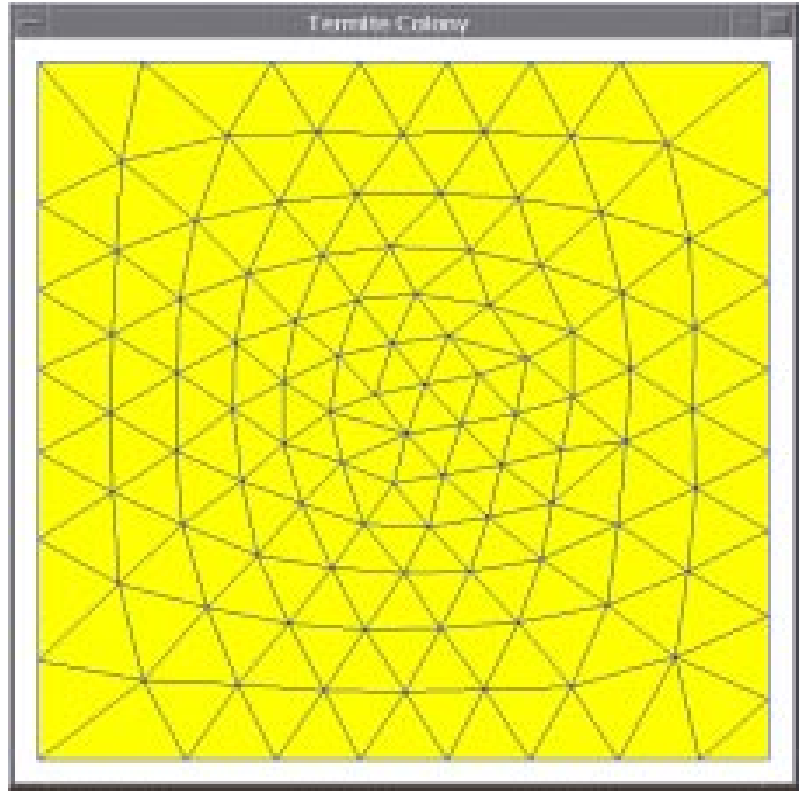

Figure 6: Meshing with Probabilistic Evolved Rules

as shown in Figures 8, 9 and 10. Fitness values are given in Tables 1 and 2 for $f_{s}$, the average size-shape metric, $f_{c}$, the average connectivity metric, $f_{t a}$, the proportion of the total domain area filled. The number of elements $e$ are also shown. The probabilistic rule set $R_{P}$ is used to mesh each domain to completion and the deterministic rule set $R_{D}$ is then run for the same number of time steps to compare results. The density functions and boundary points defining each domain are given below.

\section{Domain $\mathrm{D}_{1}$}

$$
\begin{aligned}
d= & 150 /\left(x^{2} y^{2}+0.05\right) \\
D= & \{(0.125,0.125),(0.875,0.125) \\
& (0.875,0.875),(0.125,0.875)\}
\end{aligned}
$$

\section{Domain $\mathrm{D}_{2}$}

$$
\begin{aligned}
& d=\left\{\begin{array}{l}
1200 x y+50 \\
1200(1-x) y+50 \\
1200(1-x)(1-y)+50 \\
1200 x(1-y)+50
\end{array}\right. \\
& D=\{(0.1,0.15),(0.3,0.15),(0.4,0.45), \\
& (0.6,0.45),(0.7,0.15),(0.9,0.15) \text {, } \\
& (0.9,0.85),(0.7,0.85),(0.6,0.55) \text {, } \\
& (0.4,0.55),(0.3,0.85),(0.1,0.85)\}
\end{aligned}
$$$$
\text { if } x \leq 0.5, y \leq 0.5
$$$$
\text { if } x>0.5, y \leq 0.5
$$$$
\text { if } x>0.5, y>0.5
$$

\section{Domain $\mathrm{D}_{3}$}

$$
d= \begin{cases}\frac{150}{((0.5-x)+(0.5-y))^{3}} & \text { if } x \leq 0.45, y \leq 0.45 \\ \frac{150}{((x-0.4)+(0.5-y))^{3}} & \text { if } x>0.45, y \leq 0.45 \\ \frac{150}{((x-0.4)+(y-0.4))^{3}} & \text { if } x>0.45, y>0.45 \\ \frac{150}{((0.5-x)+(y-0.4))^{3}} & \text { if } x \leq 0.45, y>0.45\end{cases}
$$

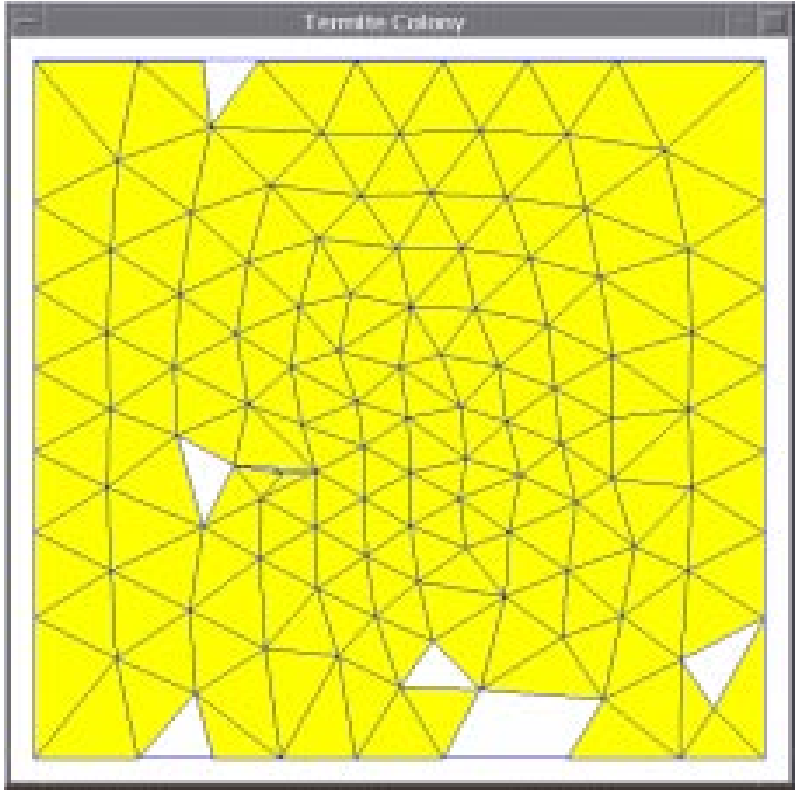

Figure 7: Meshing with Deterministic Evolved Rules

$$
\begin{aligned}
D= & \{\{(0.125,0.425),(0.225,0.408), \\
& (0.275,0.385),(0.325,0.349),(0.375,0.291), \\
& (0.4,0.24),(0.41,0.2),(0.425,0.125), \\
& (0.875,0.125),(0.875,0.875),(0.125,0.875)\} \\
& \{(0.4,0.4),(0.5,0.4),(0.5,0.5),(0.4,0.4)\}\}
\end{aligned}
$$

\begin{tabular}{|c|c|c|c|c|}
\hline Rule Set & $R_{P}$ & $R_{P}$ & $R_{P}$ & $R_{P}$ \\
\hline Domain & $f_{s}$ & $f_{c}$ & $f_{t a}$ & $e$ \\
\hline$D_{t}$ & 0.88 & 0.93 & 1.00 & 196 \\
\hline$D_{1}$ & 0.89 & 0.96 & 1.00 & 1147 \\
\hline$D_{2}$ & 0.86 & 0.86 & 1.00 & 161 \\
\hline$D_{3}$ & 0.87 & 0.96 & 1.00 & 1124 \\
\hline
\end{tabular}

Table 1: Mesh Quality for New Domains (Prob)

\begin{tabular}{|c|c|c|c|c|c|}
\hline Rule Set & $R_{D}$ & $R_{D}$ & $R_{D}$ & $R_{D}$ & $R_{P}$ and $R_{D}$ \\
\hline Domain & $f_{s}$ & $f_{c}$ & $f_{t a}$ & $e$ & Time Steps \\
\hline$D_{t}$ & 0.80 & 0.89 & 0.91 & 196 & 800 \\
\hline$D_{1}$ & 0.82 & 0.90 & 0.92 & 1014 & 7521 \\
\hline$D_{2}$ & 0.77 & 0.75 & 0.93 & 159 & 874 \\
\hline$D_{3}$ & 0.83 & 0.89 & 0.82 & 978 & 9386 \\
\hline
\end{tabular}

Table 2: Mesh Quality for New Domains (Det)

\section{Discussion}

The approach outlined in this paper evolves good meshes because the task is distributed across the domain so that previous building activity can be modified in response to new configurations in the local environment. For example Merge is 


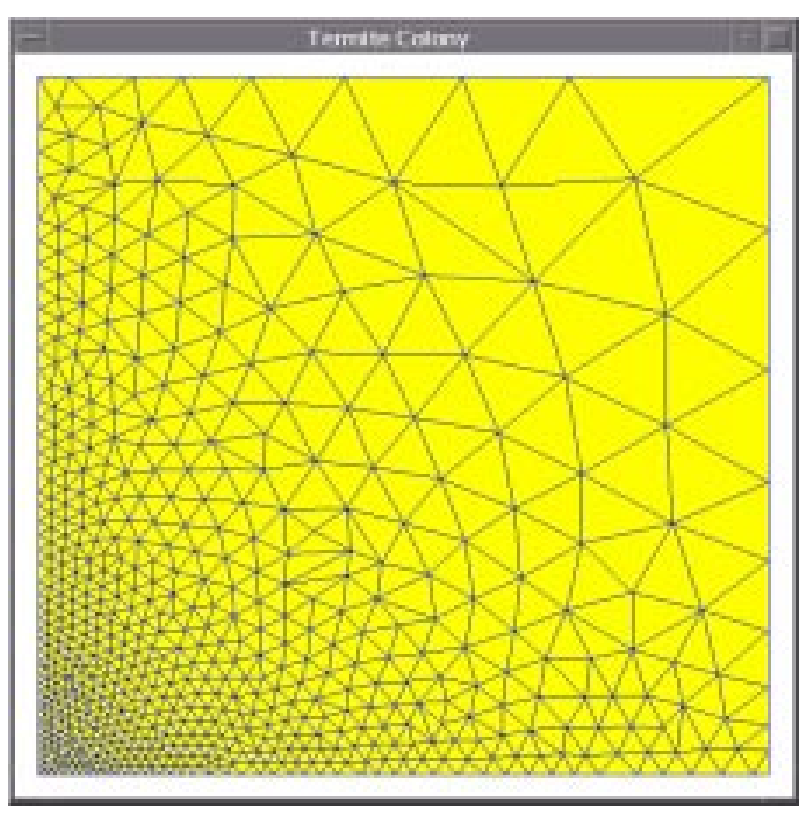

Figure 8: Probabilistic Rule Set Generalizing to Domain1

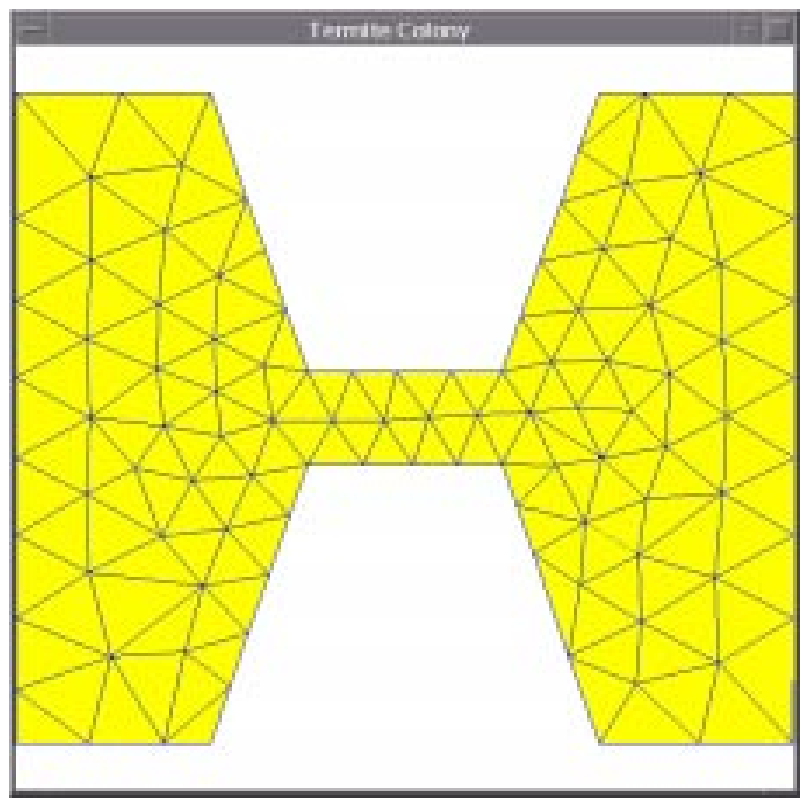

Figure 9: Probabilistic Rule Set Generalizing to Domain2

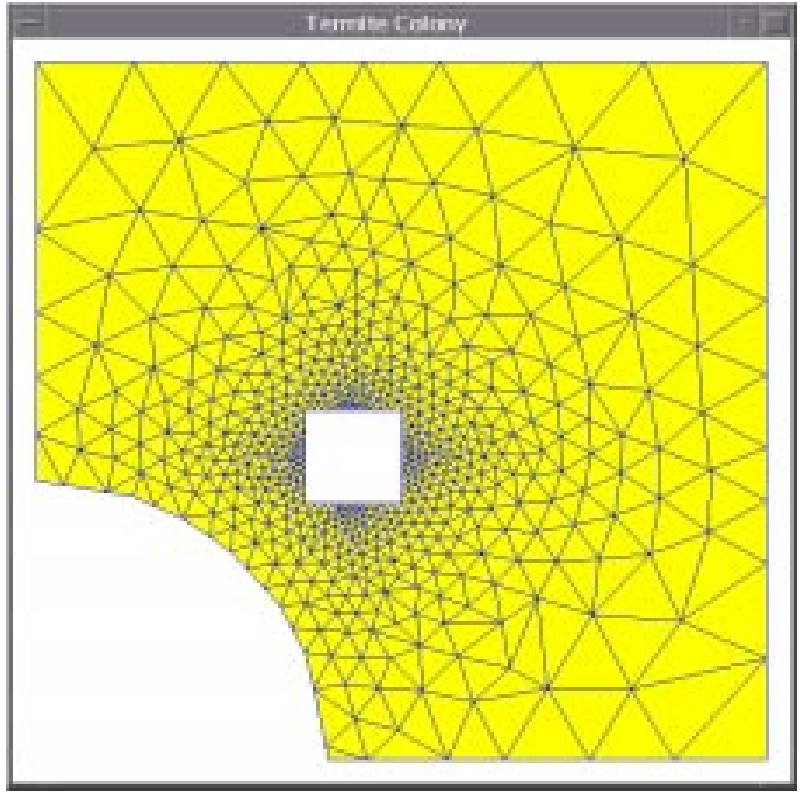

Figure 10: Probabilistic Rule Set Generalizing to Domain3

used to connect newly grown elements to existing elements. Similarly Morph is used to improve the average fitness of connected elements in the local environment and Delete eradicates connected elements in the local environment if the average fitness is below a given value. Meshes evolved by Saitou and Jakiela [4] show poorly shaped elements around boundaries. To overcome such problems our method concentrates first on boundary growth, by setting a root on each line segment this is distributed and generation takes place on all segments simultaneously. The Join operation is also used here to modify elements which have become too small and can be joined into one element of a more useful size. Boundary Growth produces well shaped elements around boundaries as can be seen from Figures 3 and 4 which shows root placement and boundary growth around a domain.

Why does this approach generalize better than the sequential approach which needs to evolve a new set of rules for each domain? The boundary growth produces well shaped elements around the boundaries and the extra operations make mesh generation much more flexible (as discussed above). Also the look condition uses information in the local environment which is directly relevant to building activity. For example $\mathrm{Can}_{g_{l}}$ is directly related to the grow left operation. Hence condition-action pairs evolved for use on one domain can be re-used in new situations. This can be seen from Tables 1 and 2 , both the probabilistic and deterministic rules show similar results on new meshes to those on the test meshes.

The condition in the sequential algorithm uses the distance to the nearest boundary/element in eight radial directions. One condition can therefore represent rather different configurations of elements and is not so relevant to the possible meshing operations. However the Probabilistic rule set generates much fitter meshes and generalizes better to new 
domains. This is because each probabilistic rule encodes a set of possibilities rather than just one action. Hence the set of possible actions are greatly increased and a generation is less likely to get stuck in a configuration where no building activity is occurring, e.g. if all conditions evoke Morph but the domain is not yet filled. Eventually another action will be evoked leading to a new condition and hence building activity can resume.

Standard methods of mesh generation construct a system of points at the required density and use this for triangulation. Examples include Delauney [7] which uses a Dirichlet Tesselation for triangulation and Quadtree methods [8] which use an underlying grid to construct elements of the required shape and size and physically based generation [9]. Our method distributes the task of mesh generation so that element creation can take place simultaneously over all parts of the domain and previous elements can be modified in response to changes in the local environment due to the creation of new elements. Similarly, our technique can respond to other changes in the environment, such as changes in the domain boundaries. Because building activity is in response to the state of the local environment the domain does not have to be fixed before mesh generation can begin. This makes our approach particularly suitable for applications such as injection moulding where the mesh must 'grow' with the domain.

Other self-organising techniques for mesh generation include the use of Kohonen neural networks [10]. Such a method is described in [11] which is based on self-organising feature maps found in sensory pathways in the brain. One disadvantage of this approach is the length of time required to train the network and retraining is necessary for each domain.

\section{Conclusion}

In this article, a distributed approach to finite element mesh generation is proposed. Using only stimuli from the local environment a colony of termite-like agents construct a mesh. To coordinate the activity of the group, no central control mechanism is required. Neither is any a priori knowledge of the global domain needed. The rule sets evolved generate high quality meshes and generalize well to new domains and density functions, with the probabilistic rule set outperforming the deterministic. Applications such as adaptive remeshing and injection moulding which involve changing environments can easily be dealt with by this method as building activity is in response to the state of the current local environment.

\section{Acknowledgments}

The first author was supported by a University of Wales Validation Unit studentship during the pursuit of this research.

\section{Bibliography}

[1] P. Grassé, "La reconstruction du nid et les coordinations interindividuelles; la théorie de la stigmergie," Insectes Sociaux, vol. 35, pp. 41-84, 1959.

[2] E. Bonabeau, G. Theraulez, E. Arpin, and E. Sardet, "The building behaviour of lattice swarms," in Artificial Life Four, July 1995.

[3] J. Denoubourg, G. Theraulaz, and R. Beckers, "Swarmmade architectures," in First European Conference on Artificial Life, July 1992.

[4] K. Saitou and M. Jakiela, "Meshing of engineering domains by meitotic cell division," in Artificial Life Four, July 1995 .

[5] D. Goldberg, Genetic Algorithms in search, optimization and machine learning. Addison-Wesley, 1989.

[6] M. Mitchell, An Introduction to Genetic Algorithms. MIT Press, 1996.

[7] J. Cavendish, "Automatic triangulation of arbitary triangular domains for the finite element method," International Journal of Numerical Methods in Engineering, vol. 8, pp. 679-697, 1974.

[8] M. Yerry and M. Shepard, "A modified-quadtree approach to finite element mesh generation," Computer Graphics Applications, vol. 3, pp. 39-46, 1983.

[9] K. Shimada and D. Gossard, "Computational methods for physically based fe mesh generation," in Human Aspects in Computer Integrated Manufacturing, July 1992.

[10] T. Kohonen, Self-Organizing and Associative Memory. Springer-Verlag, 1987.

[11] C. Ahn, S. Lee, H. Lee, and S. Lee, "A self-organizing neural network approach for automatic mesh generation," IEEE Trans Magn., vol. 27, no. 5, pp. 4201-4204, 1991. 